Research Article

\title{
The harmonic index for trees with given domination number
}

\author{
Xipeng $\mathrm{Hu}^{1,2}$, Lingping Zhong ${ }^{1,2, *}$ \\ ${ }^{1}$ Department of Mathematics, Nanjing University of Aeronautics and Astronautics, Nanjing 210016, China \\ ${ }^{2}$ Key Laboratory of Mathematical Modelling and High Performance Computing of Air Vehicles (NUAA), MIIT, Nanjing 210016, China
}

(Received: 18 October 2021. Accepted: 14 November 2021. Published online: 29 November 2021.)

(c) 2021 the authors. This is an open access article under the CC BY (International 4.0) license (www.creativecommons.org/licenses/by/4.0/).

\begin{abstract}
For a graph $G$, let $u v$ be an edge of $G$. The weight of $u v$ is defined as $2(d(u)+d(v))^{-1}$, where $d(u)$ and $d(v)$ denote the degree of the vertices $u$ and $v$, respectively. In this paper, we consider the harmonic index $H(G)$ which is the sum of weights of all edges of $G$, and obtain the extremal values of trees in terms of the order and domination number of $G$. We also characterize the extremal trees.
\end{abstract}

Keywords: harmonic index; domination number; tree.

2020 Mathematics Subject Classification: 05C07, 05C35, 05 C92.

\section{Introduction}

Let $G=(V, E)$ be a simple connected graph with vertex set $V$ and edge set $E$. A tree is a graph $G$ with $n$ vertices and $n-1$ edges. Denote by $P_{n}$ and $S_{n}$ the path and the star on $n$ vertices, respectively.

The set of neighbours of $v$ is defined as $N(v)=\{u \in V(G) \mid u v \in E(G)\}$, and $d(v)=|N(v)|$ is called the degree of $v$. If $d(v)=1$, then $v$ is a pendant vertex and its neighbour is called a support vertex. The maximum vertex degree of a graph $G$ is denoted by $\Delta(G)=\max \{d(v) \mid v \in V(G)\}$. For a vertex set $D \subseteq V(G)$, if every vertex in $V(G) \backslash D$ is adjacent to at least one vertex in $D$, then we say $D$ is a dominating set of $G$. The domination number of $G$, denoted by $\gamma(G)$, is the minimum cardinality of $D$. Let $T(n, \gamma)$ be the set of trees with $n$ vertices and domination number $\gamma$. The diameter of a tree is the number of edges of the longest path between any two pendant vertices. If the diameter of a tree $T$ is $d$, then we call $v_{0} v_{1} \cdots v_{d}$ a diameter path of $T$, denoted by $\operatorname{diam}(T)$. For the terminology and notations not defined here, we refer the readers to [3].

In 2009 and 2010, Trinajstić and Zhou proposed sum-connectivity index [12] and general sum-connectivity index [13], respectively. Later, they obtained lots of results on general sum-connectivity index $[6,9,11,12]$. As far as we know, the harmonic index

$$
H(G)=\sum_{u v \in E(G)} \frac{2}{d(u)+d(v)}
$$

was first appeared in [7]. In fact, it can be treated as a special case of general sum-connectivity index. In chemical properties, the harmonic index exhibits a strong correlation to the molecules such as boiling point, structure sensitivity and abruptness [8]. In mathematics, the relations between the harmonic index and other graph invariants are considered, such as matching number, chromatic number and the girth of a graph [1,5,10,11,14]. Recently, the domination number is studied in connection with some other vertex-degree-based topological indices [2,4]. In the following, we mainly study the connection between the harmonic index and domination number.

\section{Bounds for the harmonic index on $T(n, \gamma)$}

Definition 2.1. Let $T_{n, \gamma} \in T(n, \gamma)$ and it is obtained from the star $S_{n-\gamma+1}$ by attaching a pendant edge to its $\gamma-1$ pendant vertices, respectively. For any $T \in T(n, \gamma)$, if $\Delta(T)=n-\gamma$, then $T \cong T_{n, \gamma}$.

To simplify the calculations, we take $f(n, \gamma)=\frac{2}{5} n+\frac{3}{10} \gamma-\frac{1}{6}-\frac{1}{30}(n-3 \gamma)=\frac{11}{30} n+\frac{2}{5} \gamma-\frac{1}{6}$.

Lemma 2.1. Let $T \in T(n, \gamma)$. Suppose that there exists a vertex $v \in V(T)$ such that $d(v)=i \geq 3, N(v)=\left\{u_{1}, u_{2}, \cdots u_{i}\right\}$, $d\left(u_{i}\right)=j \geq 2$ and $d\left(u_{k}\right)=1$ for all $k \in\{1,2, \cdots, i-1\}$. Set $T^{\prime}=T-u_{1}$, if $H\left(T^{\prime}\right) \leq f(n-1, \gamma)$, then $H(T)<f(n, \gamma)$. 


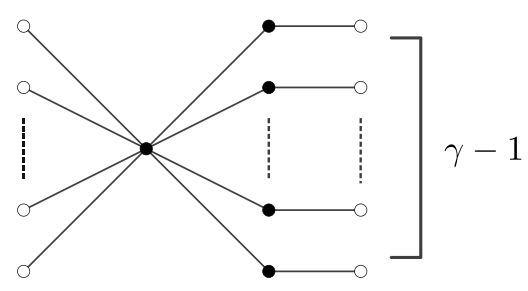

Figure 1: The graph $T_{n, \gamma}$.

Proof. Obviously, the domination number of $T^{\prime}$ is also $\gamma$, so we have

$$
\begin{aligned}
H(T) & =H\left(T^{\prime}\right)+\frac{4}{i(i+1)}-\frac{2}{(i+j)(i+j-1)} \\
& \leq \frac{2}{5}(n-1)+\frac{3}{10} \gamma-\frac{1}{6}-\frac{1}{30}(n-1-3 \gamma)+\frac{4}{i(i+1)} \\
& =f(n, \gamma)-\frac{11}{30}+\frac{4}{i(i+1)} \\
& <f(n, \gamma),
\end{aligned}
$$

since $-\frac{11}{30}+\frac{4}{i(i+1)}<0$ for any $i \geq 3$. We complete the proof.

Theorem 2.1. Let $T \in T(n, \gamma), n \geq 3$ then $H(T) \leq f(n, \gamma)$.

Proof. For $n=3,4$, we have

$$
H\left(P_{3}\right)=\frac{4}{3}=f(3,1), \quad H\left(P_{4}\right)=\frac{11}{6}<f(4,2) \quad \text { and } \quad H\left(S_{4}\right)=\frac{3}{2}<f(4,1) .
$$

Now, suppose the result is true for any tree with $n-1$ vertices and then we consider the trees of order $n$. We take a diameter path $\operatorname{diam}(T)=v_{0} v_{1} \cdots v_{d}$ in $T$. By Lemma 2.1, we can assume that $d\left(v_{1}\right)=2$. Thus, we only need to discuss the following two cases.

Case 1. $d\left(v_{2}\right)=m \geq 3$. Denote $N\left(v_{2}\right)=\left\{v_{1}, v_{3}, w_{1}, w_{2}, \cdots, w_{m-2}\right\}, d\left(w_{l}\right)=s_{l} \leq 2$ and $d\left(v_{3}\right)=k$. Then we take $T^{\prime \prime}=T-\left\{v_{0}, v_{1}\right\}$. It is clear that there exists a dominating set $D$ in $T$ such that $v_{1} \in D(T)$ and $v_{2} \in N\left(D \backslash\left\{v_{1}\right\}\right)$, which implies $\gamma(T)=\gamma\left(T^{\prime \prime}\right)+1$. Since

$$
-\frac{7}{15}+\frac{6}{(m+2)(m+1)}<0
$$

for any $m \geq 3$, we get

$$
\begin{aligned}
H(T) & =H\left(T^{\prime \prime}\right)-\frac{2}{m-1+k}-\sum_{l=1}^{m-2} \frac{2}{m-1+s_{l}}+\sum_{l=1}^{m-2} \frac{2}{m+s_{l}}+\frac{2}{m+2}+\frac{2}{1+2}+\frac{2}{m+k} \\
& \leq f(n, \gamma)-\frac{17}{15}-\sum_{l=1}^{m-2} \frac{2}{\left(m+s_{l}\right)\left(m-1+s_{l}\right)}+\frac{2}{2+m}+\frac{2}{3} \\
& \leq f(n, \gamma)-\frac{7}{15}+\frac{6}{(m+2)(m+1)} \\
& <f(n, \gamma) .
\end{aligned}
$$

Case 2. $d\left(v_{2}\right)=2$. Denote $N\left(v_{3}\right)=\left\{v_{2}, v_{4}, x_{1}, x_{2}, \cdots, x_{k-2}\right\}$ and $d\left(v_{4}\right)=r, d\left(x_{l}\right)=t_{l}$ for every $l \in\{1, \cdots, k-2\}$. For any $l \in\{1, \cdots, k-2\}$, if there exist two vertices $y_{1}, y_{2}$ such that $y_{1} \in N\left(x_{l}\right)$ and $y_{2} \in N\left(y_{1}\right)$, then we get a diameter path of $T$, i.e., $y_{2} y_{1} x_{l} v_{3} v_{4} \cdots v_{d}$, then by the above discussion, we can assume that $t_{l}=d\left(y_{1}\right)=2$.

Subcase 2.1. $k \geq 3$. Let $T^{\prime \prime \prime}=T-\left\{v_{1}, v_{2}, v_{3}\right\}$, then we have

$$
\begin{aligned}
H(T) & =H\left(T^{\prime \prime \prime}\right)-\frac{2}{k-1+r}-\sum_{l=1}^{k-2} \frac{2}{k-1+t_{l}}+\sum_{l=1}^{k-2} \frac{2}{k+t_{l}}+\frac{2}{k+r}+\frac{2}{k+2}+\frac{2}{2+2}+\frac{2}{2+1} \\
& \leq \frac{2}{5}(n-3)+\frac{3}{10}(\gamma-1)-\frac{1}{6}-\frac{1}{30}(n-3-3(\gamma-1))-\frac{2(k-2)}{(k+2)(k+1)}+\frac{2}{k+2}+\frac{1}{2}+\frac{2}{3}
\end{aligned}
$$




$$
\begin{aligned}
& =f(n, \gamma)-\frac{1}{3}+\frac{6}{(k+2)(k+1)} \\
& <f(n, \gamma),
\end{aligned}
$$

since $-\frac{1}{3}+\frac{6}{(k+2)(k+1)}<0$ for any $k \geq 3$.

Subcase 2.2. $k=2$. Denote $N\left(v_{4}\right)=\left\{v_{3}, a_{1}, \cdots, a_{r-1}\right\}$ and $d\left(a_{l}\right)=p_{l}$ for every $l \in\{1,2, \cdots, r-1\}$.

If $r=2$, then we consider the tree $T_{3}=T-\left\{v_{0}, v_{1}, v_{2}\right\}$, we have

$$
H(T)=H\left(T_{3}\right)-\frac{2}{1+2}+\frac{2 \times 3}{2+2}+\frac{2}{1+2} \leq \frac{2}{5}(n-3)+\frac{3}{10}(\gamma-1)-\frac{1}{6}-\frac{1}{30}(n-3 \gamma)+\frac{3}{2}=f(n, \gamma)
$$

For $r \geq 3$, if $p_{l} \leq 2$ for every $l \in\{1,2, \cdots, r-1\}$, let $T_{4}=T-\left\{v_{0}, v_{1}, v_{2}, v_{3}\right\}$, we have

$$
\begin{aligned}
H(T) & =H\left(T_{4}\right)-\sum_{l=1}^{r-1} \frac{2}{r-1+p_{l}}+\sum_{l=1}^{r-1} \frac{2}{r+p_{l}}+\frac{2}{2+r}+\frac{4}{2+2}+\frac{2}{2+1} \\
& \leq \frac{2}{5}(n-4)+\frac{3}{10}(\gamma-1)-\frac{1}{6}-\frac{1}{30}(n-4-3(\gamma-1))-\sum_{l=1}^{r-1} \frac{2}{\left(r-1+p_{l}\right)\left(r+p_{l}\right)}+\frac{2}{2+r}+\frac{5}{3} \\
& \leq f(n, \gamma)-\frac{1}{5}+\frac{4}{(r+2)(r+1)}<f(n, \gamma) .
\end{aligned}
$$

where $-\frac{1}{5}+\frac{4}{(r+2)(r+1)}<0$ for any $r \geq 3$.

Let $p_{1}=\max \left\{p_{1}, \cdots, p_{r-1}\right\} \geq 3$, and $N\left(a_{1}\right)=\left\{v_{4}, b_{1}, b_{2}, \cdots, b_{p_{1}-1}\right\}$, by the above case and Lemma 2.1 , we assume that every vertex in $N\left(b_{l}\right) \backslash\left\{a_{1}\right\}$ is a pendant vertex for any $l \in\left\{1,2, \cdots, q_{1}-1\right\}$ and $1 \leq d\left(b_{l}\right) \leq 2$. Denote by $q_{i}$ the number of vertices in $\left\{b_{1}, b_{2}, \cdots, b_{p_{1}-1}\right\}$ with degree $i$. Consider the edge $e=v_{4} a_{1}, T_{v_{4}}$ and $T_{a_{1}}$ are two components of $T-e$, which contain the vertex $v_{4}$ and $a_{1}$, respectively. Then

$$
\begin{aligned}
H(T)= & H\left(T_{v_{4}}\right)+H\left(T_{a_{1}}\right)-\sum_{l=2}^{r-1} \frac{2}{r-1+p_{l}}+\sum_{l=2}^{r-1} \frac{2}{r+p_{l}}+\frac{2}{p_{1}+r}-\frac{2}{r-1+2} \\
& +\frac{2}{r+2}-\frac{2 q_{1}}{p_{1}-1+1}+\frac{2 q_{1}}{p_{1}+1}-\frac{2 q_{2}}{p_{1}-1+2}+\frac{2 q_{2}}{p_{1}+2} \\
\leq & f(n, \gamma)-\frac{1}{6}-\frac{2(r-2)}{\left(r-1+p_{1}\right)\left(r+p_{1}\right)}+\frac{2}{p_{1}+r}-\frac{2}{1+r}+\frac{2}{2+r}-\frac{2 q_{1}}{\left(p_{1}+1\right)\left(p_{1}+2\right)}-\frac{2 q_{2}}{\left(p_{1}+1\right)\left(p_{1}+2\right)} \\
\leq & f(n, \gamma)-\frac{1}{6}+\frac{2\left(p_{1}+1\right)}{\left(p_{1}+3\right)\left(p_{1}+2\right)}-\frac{2\left(p_{1}-1\right)}{\left(p_{1}+1\right)\left(p_{1}+2\right)}-\frac{2}{(r+1)(r+2)} \\
= & f(n, \gamma)-\frac{1}{6}+\frac{8}{\left(p_{1}+1\right)\left(p_{1}+2\right)\left(p_{1}+3\right)}-\frac{2}{(r+1)(r+2)} \leq f(n, \gamma),
\end{aligned}
$$

since $-\frac{1}{6}+\frac{8}{\left(p_{1}+1\right)\left(p_{1}+2\right)\left(p_{1}+3\right)}<0$, for all $p_{1} \geq 3$.

\section{Lemma 2.2. Let}

$$
h(n, k)=\frac{k}{(n-k)(n-k+1)}-\frac{k-1}{(n-k+1)(n-k+2)},
$$

then $h\left(n, k+\frac{1}{2}\right)>h(n, k)$ and $h(n, k)>h(n+1, k)$, for any $n>2$ and $1 \leq k \leq n-2$.

Proof. First, we see that $h\left(n, k+\frac{1}{2}\right)>h(n, k)$ for any $k \leq n-2$.

$$
\begin{aligned}
h\left(n, k+\frac{1}{2}\right)-h(n, k)= & \frac{k+\frac{1}{2}}{\left(n-k-\frac{1}{2}\right)\left(n-k+\frac{1}{2}\right)}-\frac{k-\frac{1}{2}}{\left(n-k+\frac{1}{2}\right)\left(n-k+\frac{3}{2}\right)} \\
= & k\left(\frac{1}{\left(n-k-\frac{1}{2}\right)\left(n-k+\frac{1}{2}\right)}-\frac{1}{\left(n-k+\frac{1}{2}\right)\left(n-k+\frac{3}{2}\right)}\right. \\
& \left.-\frac{1}{(n-k)(n-k+1)}+\frac{1}{(n-k+1)(n-k+2)}\right) \\
& +\frac{1}{2\left(n-k-\frac{1}{2}\right)\left(n-k+\frac{1}{2}\right)}+\frac{1}{2\left(n-k+\frac{1}{2}\right)\left(n-k+\frac{3}{2}\right)}-\frac{1}{(n-k+1)(n-k+2)}
\end{aligned}
$$

Since

$$
\frac{1}{\left(x-\frac{1}{2}\right)\left(x+\frac{1}{2}\right)}-\frac{1}{\left(x+\frac{1}{2}\right)\left(x+\frac{3}{2}\right)}-\frac{1}{x(x+1)}+\frac{1}{(x+1)(x+2)}=\frac{8}{\left(x-\frac{1}{2}\right)\left(x+\frac{1}{2}\right)\left(x+\frac{3}{2}\right)}-\frac{2}{x(x+1)(x+2)}>0
$$


for all $x>0$, so

$$
\frac{1}{\left(n-k-\frac{1}{2}\right)\left(n-k+\frac{1}{2}\right)}-\frac{1}{\left(n-k+\frac{1}{2}\right)\left(n-k+\frac{3}{2}\right)}-\frac{1}{(n-k)(n-k+1)}+\frac{1}{(n-k+1)(n-k+2)}>0,
$$

for any $n-k \geq 2$.

$$
\frac{1}{2\left(x-\frac{1}{2}\right)\left(x+\frac{1}{2}\right)}+\frac{1}{2\left(x+\frac{1}{2}\right)\left(x+\frac{3}{2}\right)}-\frac{1}{(x+1)(x+2)}=\frac{2}{\left(x-\frac{1}{2}\right)\left(x+\frac{3}{2}\right)}-\frac{1}{(x+1)(x+2)}>0,
$$

for all $x>0$, so

$$
\frac{1}{2\left(n-k-\frac{1}{2}\right)\left(n-k+\frac{1}{2}\right)}+\frac{1}{2\left(n-k+\frac{1}{2}\right)\left(n-k+\frac{3}{2}\right)}-\frac{1}{(n-k+1)(n-k+2)}>0,
$$

thus $h\left(n, k+\frac{1}{2}\right)>h(n, k)$.

Since

$$
\begin{aligned}
h(n, k)-h(n+1, k) & =\frac{k}{(n-k)(n-k+1)}-\frac{k-1}{(n-k+1)(n-k+2)}-\frac{k}{(n-k+1)(n-k+2)}+\frac{k-1}{(n-k+2)(n-k+3)} \\
& =\frac{6 k}{(n-k)(n-k+1)(n-k+2)(n-k+3)}+\frac{2}{(n-k+1)(n-k+2)(n-k+3)}
\end{aligned}
$$

is a positive function for any $n-k \geq 2$, hence we have the required inequality.

Theorem 2.2. Let $T \in T(n, \gamma)$, then

$$
H(T) \geq 2\left(\frac{n-2 \gamma+1}{n-\gamma+1}+(\gamma-1)\left(\frac{1}{3}+\frac{1}{n-\gamma+2}\right)\right) .
$$

Proof. We use induction on the number of vertices. It's easy to check that the inequality holds for the star $S_{4}$ and the path $P_{4}$. Thus we can suppose it is true for any tree with $n-1$ vertices. We denote

$$
g(n, \gamma)=2\left(\frac{n-2 \gamma+1}{n-\gamma+1}+(\gamma-1)\left(\frac{1}{3}+\frac{1}{n-\gamma+2}\right)\right) .
$$

Analogously, we suppose that $\operatorname{diam}(G)=v_{0} v_{1} \cdots v_{d}$ is a diameter path in $T$. Let us take $N\left(v_{1}\right)=\left\{v_{0}, v_{2}, u_{1}, \cdots, u_{i-2}\right\}$, $N\left(v_{2}\right)=\left\{v_{1}, v_{3}, w_{1}, \cdots w_{m-2}\right\}$ and $d\left(w_{l}\right)=s_{l}$, for every $l \in\{1, \cdots, m-2\}$. Take $T^{\prime}=T-v_{0}$ for consideration and we study the following two cases.

Case 1. If $\gamma\left(T^{\prime}\right)=\gamma(T)$, then

$$
\begin{aligned}
H(T) & =H\left(T^{\prime}\right)-\frac{2(i-2)}{i-1+1}-\frac{2}{i-1+m}+\frac{2(i-1)}{i+1}+\frac{2}{2+m} \\
& \geq 2\left(\frac{n-2 \gamma}{n-\gamma}+(\gamma-1)\left(\frac{1}{n-\gamma+1}+\frac{1}{3}\right)\right)+\frac{4}{i(i+1)}-\frac{2}{(i+m)(i+m-1)} \\
& =g(n, \gamma)+2\left(\frac{-\gamma}{(n-\gamma)(n-\gamma+1)}+\frac{\gamma-1}{(n-\gamma+1)(n-\gamma+2)}\right)+\frac{4}{i(i+1)}-\frac{2}{(i+2)(i+1)} .
\end{aligned}
$$

If $n=i+2$, then we have the graph shown in Figure 1 with $m=1$, so we may assume $n \geq i+3$. Since $\gamma \leq \frac{n-i+2}{2}$ and $n \geq i+3$, by Lemma 2.2 we know that

$$
\frac{\gamma}{(n-\gamma)(n-\gamma+1)}-\frac{\gamma-1}{(n-\gamma+1)(n-\gamma+2)} \leq \frac{\frac{n-i+2}{2}}{\left(\frac{n}{2}+\frac{i}{2}-1\right)\left(\frac{n}{2}+\frac{i}{2}\right)}+\frac{\frac{n-i}{2}}{\left(\frac{n}{2}+\frac{i}{2}+1\right)} \leq \frac{5}{2\left(i+\frac{1}{2}\right)\left(i+\frac{3}{2}\right)}-\frac{3}{2\left(i+\frac{3}{2}\right)\left(i+\frac{5}{2}\right)},
$$

then

$$
\begin{aligned}
& -2\left(\frac{\gamma}{(n-\gamma)(n-\gamma+1)}-\frac{\gamma-1}{(n-\gamma+1)(n-\gamma+2)}\right)+\frac{4}{i(i+1)}-\frac{2}{(i+2)(i+1)} \\
& \geq \frac{-5}{\left(i+\frac{1}{2}\right)\left(i+\frac{3}{2}\right)}+\frac{3}{\left(i+\frac{3}{2}\right)\left(i+\frac{5}{2}\right)}+\frac{4}{i(i+1)}-\frac{2}{(i+2)(i+1)}=\frac{6\left(14 i^{2}+37 i+20\right)}{i(i+1)(i+2)(2 i+1)(2 i+3)(2 i+5)}>0,
\end{aligned}
$$

therefore, $H(T)>g(n, \gamma)$.

Case 2. If $\gamma\left(T^{\prime}\right)=\gamma(T)-1$, then $i=2$ and there exists a minimum dominating set $D$ of $T$ such that $v_{2} \in D$. Therefore,

$$
H(T)=H\left(T^{\prime}\right)-\frac{2}{1+m}+\frac{2}{2+m}+\frac{2}{1+2} \geq 2\left(\frac{n-2 \gamma+2}{n-\gamma+1}+(\gamma-2)\left(\frac{1}{3}+\frac{1}{n-\gamma+2}\right)\right)-\frac{2}{(m+1)(m+2)}+\frac{2}{3}
$$




$$
=g(n, \gamma)+2\left(\frac{1}{(n-\gamma+1)(n-\gamma+2)}-\frac{1}{(m+1)(m+2)}\right) .
$$

If $m \geq n-\gamma$, it's done, but this only happens when $\gamma=n-m$. Since $n-m=\gamma \leq \frac{n}{2}$, we have $n \leq 2 m$ (see the graph in Figure 1).

If $m \leq n-\gamma-1$, we denote $N\left(v_{2}\right)=\left\{v_{1}, v_{3}, w_{1}, w_{2}, \cdots, w_{m-2}\right\}$ and $d\left(w_{l}\right)=s_{l}$ for any $l \in\{1,2, \cdots, m-2\}$. We assume that $s_{l}=1$ or $w_{l}$ is a support vertex with $s_{l}=2$ for any $l \in\{1,2, \cdots, m-2\}$. If $v_{3}$ is a vertex of degree 1 or a support vertex of degree 2, then the graph is one of the trees shown in Figure 1, which satisfies the equality. For otherwise, we have $s_{1}=\cdots=s_{r_{1}}=1, s_{r_{1}+1}=\cdots=s_{r_{1}+r_{2}}=2$ and $r_{1}+r_{2}=m-2$, then we consider the following situation.

If $d\left(w_{1}\right)=1$, set $T_{1}=T-w_{1}$, then $\gamma\left(T_{1}\right)=\gamma(T)$. Since

$$
\gamma-\left(2+r_{2}\right) \leq \frac{n-\left(m+1+r_{2}\right)}{2},
$$

we have

$$
\gamma \leq \frac{n-r_{1}+1}{2}
$$

and consequently,

$$
\begin{aligned}
H(T) & =H\left(T_{1}\right)-\frac{2\left(r_{1}-1\right)}{m-1+1}+\frac{2\left(r_{1}-1\right)}{j+1}-\frac{2\left(r_{2}+1\right)}{m-1+2}+\frac{2\left(r_{2}+1\right)}{m+2}-\frac{2}{d\left(v_{3}\right)+m-1}+\frac{2}{d\left(v_{3}\right)+m}+\frac{2}{m+1} \\
& \geq 2\left(\frac{n-2 \gamma}{n-\gamma}+(\gamma-1)\left(\frac{1}{3}+\frac{1}{n-\gamma+1}\right)\right)-\frac{2\left(r_{1}-1\right)}{m(m+1)}-\frac{2\left(r_{2}+1\right)}{(m+1)(m+2)}-\frac{2}{(m+1)(m+2)}+\frac{2}{m+1} \\
& =g(n, \gamma)-\frac{2 \gamma}{(n-\gamma)(n-\gamma+1)}+\frac{2(\gamma-1)}{(n-\gamma+1)(n-\gamma+2)}+\frac{2\left(m-r_{1}+1\right)}{m(m+1)}-\frac{2\left(m-r_{1}\right)}{(m+1)(m+2)} .
\end{aligned}
$$

We know that there exists $r \geq 0$ such that $n=r_{1}+2 r_{2}+5+r$, so

$$
\gamma \leq \frac{\left(r_{1}+2 r_{2}+5+r\right)-r_{1}+1}{2}=r_{2}+3+\frac{r}{2}
$$

and by Lemma 2.2, we can conclude that $h(n, \gamma) \leq h\left(r_{1}+2 r_{2}+5+r, r_{2}+3+\frac{r}{2}\right)$, which means

$$
\begin{aligned}
-\frac{2 \gamma}{(n-\gamma)(n-\gamma+1)}+\frac{2(\gamma-1)}{(n-\gamma+1)(n-\gamma+2)} & \geq \frac{-2\left(r_{2}+3+\frac{r}{2}\right)}{\left(m+\frac{r}{2}\right)\left(m+\frac{r}{2}+1\right)}+\frac{2\left(r_{2}+2+\frac{r}{2}\right)}{\left(m+\frac{r}{2}+1\right)\left(m+\frac{r}{2}+2\right)} \\
& =-\frac{2\left(m+\frac{r}{2}+1-r_{1}\right)}{\left(m+\frac{r}{2}\right)\left(m+\frac{r}{2}+1\right)}+\frac{2\left(m+\frac{r}{2}-r_{1}\right)}{\left(m+\frac{r}{2}+1\right)\left(m+\frac{r}{2}+2\right)} .
\end{aligned}
$$

For

if $r_{1} \leq x$, then for any $x \geq 2$,

$$
f(x)=\frac{x-r_{1}+1}{x(x+1)}-\frac{x-r_{1}}{(x+1)(x+2)},
$$

$$
\begin{aligned}
f^{\prime}(x) & =-\frac{4+12 x+15 x^{2}+6 x^{3}-2 r_{1}\left(2+6 x+3 x^{2}\right)}{x^{2}(1+x)^{2}(2+x)^{2}} \\
& \leq-\frac{4+12 x+15 x^{2}+6 x^{3}-2 x\left(2+6 x+3 x^{2}\right)}{x^{2}(1+x)^{2}(2+x)^{2}}=\frac{-2-3 x}{x^{2}(1+x)^{2}(2+x)}<0 .
\end{aligned}
$$

Therefore, $f(x)$ is a decreasing function for any $x \geq r_{1}$. Since $m+\frac{r}{2} \geq m \geq r_{1}$, so if $r=0$, then the graph is one of the graph shown in Figure 1, for otherwise, we have $f\left(m+\frac{r}{2}\right)<f(m)$, which implies that

$$
\frac{2\left(m-r_{1}+1\right)}{m(m+1)}-\frac{2\left(m-r_{1}\right)}{(m+1)(m+2)} \geq \frac{2\left(m+\frac{r}{2}+1-r_{1}\right)}{\left(m+\frac{r}{2}\right)\left(m+\frac{r}{2}+1\right)}+\frac{2\left(m+\frac{r}{2}-r_{1}\right)}{\left(m+\frac{r}{2}+1\right)\left(m+\frac{r}{2}+2\right)},
$$

consequently, $H(T)>g(n, \gamma)$.

\section{Extremal trees for the harmonic index on $T(n, \gamma)$}

Let $\mathscr{H}$ be a set of trees with $P_{3 k} \in \mathscr{H}$, where $k$ is a positive integer number, then we construct new graphs in family $\mathscr{H}$ in the following two ways (see Figure 2).

(i) If $T^{\prime} \in \mathscr{H}$ satisfies that there exists a vertex $v$ belongs to a minimum dominating set of $T^{\prime}$ such that $N(v)=\left\{u_{1}, u_{2}\right\}$, $d\left(u_{1}\right)=d\left(u_{2}\right)=2$, then attach a path of length $3 t+1$ to the vertex $v$, we get a new tree $T$, which is also in $\mathscr{H}$.

(ii) If $T^{\prime} \in \mathscr{H}, v \in V\left(T^{\prime}\right)$ is a pendant vertex, then attach a path of length $3 t$ to the vertex $v$, we get a new tree $T$ in $\mathscr{H}$.

In fact, the trees constructed in (ii) are completely contained those constructed in (i), we consider the trees in two ways since it will bring convenience to the proof of the following theorem. 

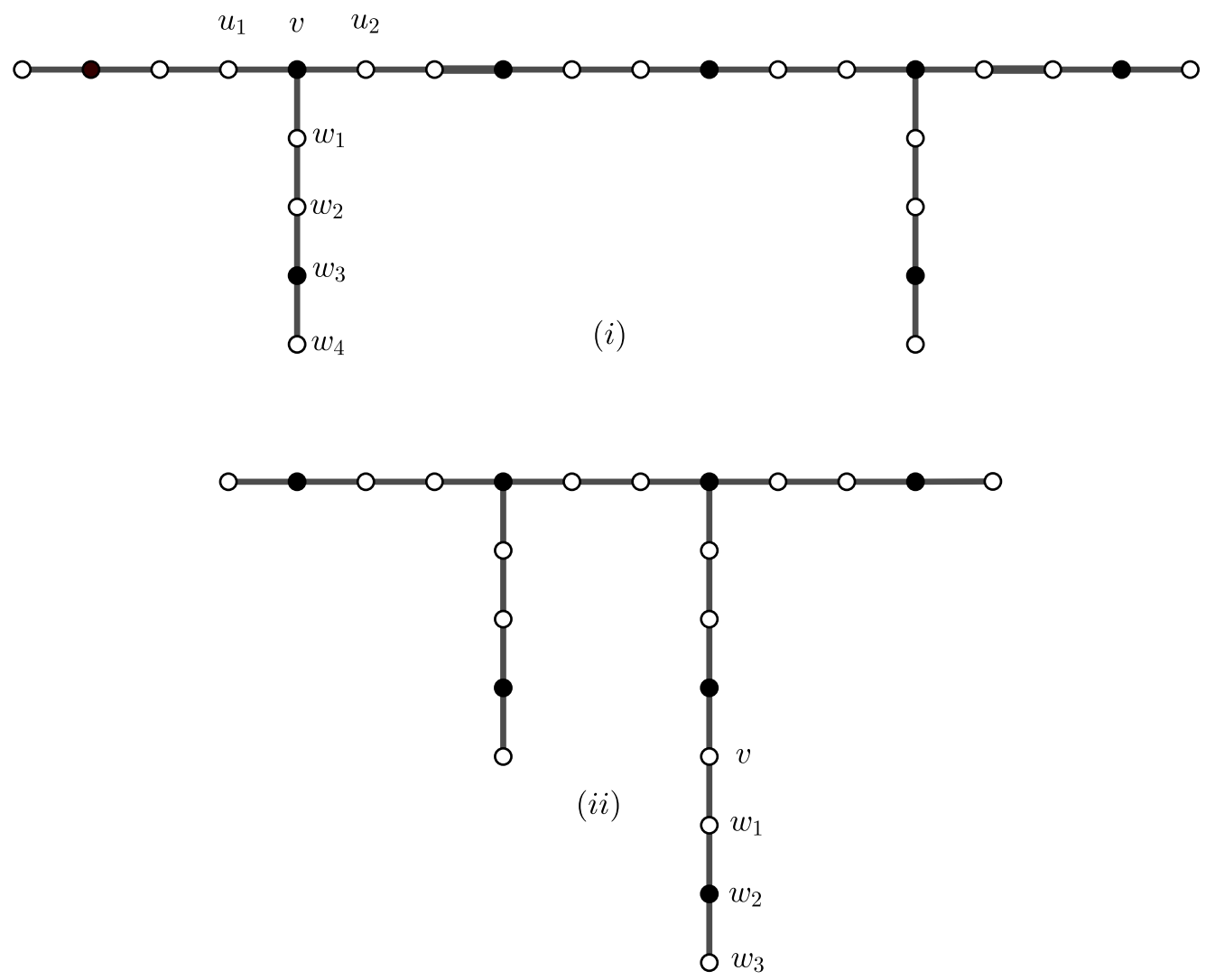

Figure 2: Two examples in graph family $\mathscr{H}$.

Lemma 3.1. If $T \in \mathscr{H}$, then $H(T)=f(n(T), \gamma(T))$.

Proof. If $T \cong P_{3 k}$, obviously it is true.

(i) We suppose that there exists $T^{\prime} \in \mathscr{H}$ satisfying $H\left(T^{\prime}\right)=f\left(n\left(T^{\prime}\right), \gamma\left(T^{\prime}\right)\right)$ and there exists $v \in V\left(T^{\prime}\right)$ such that $N(v)=\left\{u_{1}, u_{2}\right\}, d\left(u_{1}\right)=d\left(u_{2}\right)=2$, where $v$ belongs to a minimum dominating set in $T^{\prime}$. Then we attach a path of length $3 t+1$ to $v$, we have

$$
\begin{aligned}
H(T) & =H\left(T^{\prime}\right)+H(P)-2\left(\frac{2}{2+2}+\frac{1}{2+1}\right)+2\left(\frac{3}{2+3}+\frac{1}{2+2}\right) \\
& =\frac{2}{5} n\left(T^{\prime}\right)+\frac{3}{10} \gamma\left(T^{\prime}\right)-\frac{1}{6}-\frac{1}{30}\left(n\left(T^{\prime}\right)-3 \gamma\left(T^{\prime}\right)\right)+\frac{2}{5}(3 t+1)+\frac{3}{10} t-\frac{1}{30}(3 t+1-3 t) \\
& =\frac{2}{5} n(T)+\frac{3}{10} \gamma(T)-\frac{1}{6}-\frac{1}{30}(n(T)-3 \gamma(T)) .
\end{aligned}
$$

(ii) If $T^{\prime} \in \mathscr{H}$ satisfies that $H\left(T^{\prime}\right)=f\left(n\left(T^{\prime}\right), \gamma\left(T^{\prime}\right)\right)$, and $T^{\prime}$ has a pendant vertex $v$, then we attach a path of $3 t$ to $v$, we obtain

$$
\begin{aligned}
H(T) & =H\left(T^{\prime}\right)+H(P)-2 \times \frac{2}{1+2}+3 \times \frac{2}{2+2} \\
& =\frac{2}{5} n\left(T^{\prime}\right)+\frac{3}{10} \gamma\left(T^{\prime}\right)-\frac{1}{6}-\frac{1}{30}\left(n\left(T^{\prime}\right)-3 \gamma\left(T^{\prime}\right)\right)+\frac{1}{6}+2\left(\frac{2}{1+2}+\frac{3 t-3}{2+2}\right) \\
& =\frac{2}{5} n(T)+\frac{3}{10} \gamma(T)-\frac{1}{6}-\frac{1}{30}(n(T)-3 \gamma(T)) .
\end{aligned}
$$

Theorem 3.1. If $T \in T(n, \gamma)$, then $H(T)=f(n(T), \gamma(T))$ if and only if $T \in \mathscr{H}$.

Proof. By Lemma 3.1, we only need to prove sufficiency. By contradiction, suppose $T^{*} \in T(n, \gamma)$ is the tree with minimum number of vertices satisfying $H\left(T^{*}\right)=f\left(n\left(T^{*}\right), \gamma\left(T^{*}\right)\right)$ and $T^{*} \notin \mathscr{H}$. Take a diameter path $\operatorname{diam}\left(T^{*}\right)=v_{0} v_{1} \cdots v_{d}$ of $T^{*}$, then by the proof of Theorem 2.1, we may assume that $d\left(v_{1}\right)=d\left(v_{2}\right)=d\left(v_{3}\right)=2, d\left(v_{4}\right)=3$ or $d\left(v_{4}\right)=2$ and $p_{1}, p_{2} \leq 2$. 
If $d\left(v_{4}\right)=3$ and $p_{1}, p_{2} \leq 2$ and we set $T_{4}=T^{*}-\left\{v_{0}, v_{1}, v_{2}, v_{3}\right\}$, then

$$
\begin{aligned}
H\left(T^{*}\right) & =H\left(T_{4}\right)-\frac{2}{2+p_{1}}-\frac{2}{2+p_{2}}+\frac{2}{3+p_{1}}+\frac{2}{3+p_{2}}+\frac{2}{5}+1+\frac{2}{3} \\
& \leq \frac{2}{5}(n-4)+\frac{3}{10}(\gamma-1)-\frac{1}{6}-\frac{1}{30}(n-4-3(\gamma-1))-\frac{2}{2+p_{1}}-\frac{2}{2+p_{2}}+\frac{2}{3+p_{1}}+\frac{2}{3+p_{2}}+\frac{2}{5}+1+\frac{2}{3} \\
& =f(n, \gamma)+\frac{1}{5}-\frac{2}{\left(p_{1}+2\right)\left(p_{1}+3\right)}-\frac{2}{\left(p_{2}+2\right)\left(p_{2}+3\right)} .
\end{aligned}
$$

If $p_{1}=1$ or $p_{2}=1$, then

$$
\frac{1}{5}-\frac{2}{\left(p_{1}+2\right)\left(p_{1}+3\right)}-\frac{2}{\left(p_{2}+2\right)\left(p_{2}+3\right)}<0,
$$

a contradiction. Otherwise $p_{1}=p_{2}=2$, then

$$
\frac{1}{5}-\frac{2}{\left(p_{1}+2\right)\left(p_{1}+3\right)}-\frac{2}{\left(p_{2}+2\right)\left(p_{2}+3\right)}=0
$$

and

$$
H\left(T_{4}\right)=\frac{2}{5} n\left(T_{4}\right)+\frac{3}{10} \gamma\left(T_{4}\right)-\frac{1}{6}-\frac{1}{30}\left(n\left(T_{4}\right)-3 \gamma\left(T_{4}\right)\right) .
$$

If $T_{4} \in \mathscr{H}$, since $v_{4}$ belongs to a minimum dominating set and $p_{1}=p_{2}=2$, then $T^{*} \in \mathscr{H}$. Therefore, $T_{4} \notin \mathscr{H}$ and we get a contradiction with the minimality of $T^{*}$.

If $d\left(v_{4}\right)=2$ and we take $T_{3}=T^{*}-\left\{v_{0}, v_{1}, v_{2}\right\}$, we have

$$
H\left(T^{*}\right)=H\left(T_{3}\right)-\frac{2}{1+2}+\frac{2 \times 3}{2+2}+\frac{2}{1+2} \leq \frac{2}{5}(n-3)+\frac{3}{10}(\gamma-1)-\frac{1}{6}-\frac{1}{30}(n-3 \gamma)+\frac{3}{2}=f(n, \gamma)
$$

thus

$$
H\left(T_{3}\right)=\frac{2}{5} n\left(T_{3}\right)+\frac{3}{10} \gamma\left(T_{3}\right)-\frac{1}{6}-\frac{1}{30}\left(n\left(T_{3}\right)-3 \gamma\left(T_{3}\right)\right) .
$$

If $T_{3} \in \mathscr{H}$, since $v_{3}$ is a pendant vertex of $T_{3}$, so $T^{*} \in \mathscr{H}$. Therefore, $T_{3} \notin \mathscr{H}$, a contradiction to the minimality of $T^{*}$.

Through the above whole discussion, we obtain the following theorem.

Theorem 3.2. If $T \in T(n, \gamma)$, then

$$
H(T)=2\left(\frac{n-2 \gamma+1}{n-\gamma+1}+(\gamma-1)\left(\frac{1}{3}+\frac{1}{n-\gamma+2}\right)\right)
$$

if and only if $T \cong T_{n, \gamma}$.

\section{Acknowledgement}

This work was partially supported by the National Natural Science Foundation of China (through grant no. 12171239) and the Postgraduate Research \& Practice Innovation Program of Nanjing University of Aeronautics and Astronautics (through grant no. xcxjh20210802).

\section{References}

[1] A. Ali, L. Zhong, I. Gutman, Harmonic index and its generalizations: extremal results and bounds, MATCH Commun. Math. Comput. Chem. 81 (2019) 249-311.

[2] S. Bermudo, J. Nápoles, J. Rada, Extremal trees for the Randić index with given domination number, Appl. Math. Comput. 375 (2020) \#125122.

[3] J. Bondy, U. Murty, Graph Theory, Springer-Verlag, London, 2008.

[4] B. Boroviánin, B. Furtula, On extremal Zagreb indices of trees with given domination number, Appl. Math. Comput. 279 (2016) $208-218$.

[5] H. Deng, S. Balachandran, S. Ayyaswamy, Y. Venkatakrishnan, On the harmonic index and chromatic number of a graph, Discrete Appl. Math. 161 (2013) 2740-2744.

[6] Z. Du, B. Zhou, N. Trinajstić, On the general sum-connectivity index of trees, Appl. Math. Lett. 24 (2011) $402-405$.

[7] S. Fajtlowicz, On conjectures of Graffiti-II, Congr. Numer. 60 (1987) 187-197.

[8] I. Gutman, Degree-based topological indices, Croat. Chem. Acta. 86 (2013) 351-361.

[9] B. Lučić, S. Nikolić, N. Trinajstić, B. Zhou, S. I. Turk, Sum-connectivity index, In: I. Gutman, B. Furtula (Eds.), Novel Molecular Structure Descriptors - Theory and Applications I, Univ. Kragujevac, Kragujevac, 2010, pp. 101-136.

[10] J. Lv, J. Li, The harmonic index of bicyclic graphs with given matching number, Util. Math. 107 (2018) $287-304$.

[11] R. Rasi, S. Sheikholeslami, I. Gutman, On harmonic inex of trees, MATCH Commun. Math. Comput. Chem. 78 (2017) $405-416$.

[12] B. Zhou, N. Trinajstić, On a novel connectivity index, J. Math. Chem. 46 (2009) 1252-1270.

[13] B. Zhou, N. Trinajstić, On general sum-connectivity index, J. Math. Chem. 47 (2010) 210-218.

[14] L. Zhong, On the harmonic index and the girth for graphs, Rom. J. Inf. Sci. Tech. 16 (2013) 253-260. 